\title{
Visualization of multiple influences on ocellar flight control in giant honeybees with the data-mining tool Viscovery SOMine
}

\author{
GERALD KASTBERGER \\ Karl-Franzens University, Graz, Austria \\ and \\ GERHARD KRANNER \\ Eudaptics Software, Vienna, Austria
}

\begin{abstract}
Viscovery SOMine is a software tool for advanced analysis and monitoring of numerical data sets. It was developed for professional use in business, industry, and science and to support dependency analysis, deviation detection, unsupervised clustering, nonlinear regression, data association, pattern recognition, and animated monitoring. Based on the concept of self-organizing maps (SOMs), it employs a robust variant of unsupervised neural networks-namely, Kohonen's Batch-SOM, which is further enhanced with a new scaling technique for speeding up the learning process. This tool provides a powerful means by which to analyze complex data sets without prior statistical knowledge. The data representation contained in the trained SOM is systematically converted to be used in a spectrum of visualization techniques, such as evaluating dependencies between components, investigating geometric properties of the data distribution, searching for clusters, or monitoring new data. We have used this software tool to analyze and visualize multiple influences of the ocellar system on free-flight behavior in giant honeybees. Occlusion of ocelli will affect orienting reactivities in relation to flight target, level of disturbance, and position of the bee in the flight chamber; it will induce phototaxis and make orienting imprecise and dependent on motivational settings. Ocelli permit the adjustment of orienting strategies to environmental demands by enforcing abilities such as centering or flight kinetics and by providing independent control of posture and flight course.
\end{abstract}

Data sets collected from biological, psychological, and social experiments or data taken from social, economic, or marketing systems often contain hidden information that is difficult to extract. This is the starting point for a number of analysis methods, commonly known by the term data mining. Widely used traditional methods, such as statistical algorithms, mainly reproduce dependencies within data in a limited way, since they are mostly based on linear principles and a priori assumptions. Such methods imply that it would be extremely difficult to interactively explore high-dimensional, complex data distributions and their underlying nonlinear relationships.

Newer models, such as supervised neural network techniques, impose high demands on tuning the underlying algorithms (e.g., in order to design the network topology), which very often demand much effort and a timeconsuming trial-and-error process, owing to the complexity of real-world applications. On the other hand, statistical

The investigation of the ocellar role in flight control was supported by Austrian Scientific Foundation Grant P8746-BIO. The second author has a financial interest in the software described in this article. The authors thank Elisabeth Jaquemar for final improvements of the English. Correspondence concerning this article should be addressed to G. Kastberger, Institute of Zoology, Department of Neurobiology, KarlFranzens University, A8010 Graz, Austria (e-mail: gerald.kastberger@ kfunigraz.ac.at). tools have the common inherent weakness of lacking a visualization that would make possible quick and intuitive information retrieval.

The concept of self-organizing maps (SOMs) provides an alternative that is very robust with respect to setting algorithm parameters and that is purely data driven. The method is characterized by the qualifier adaptive, since the self-organizing map adapts to the shape of any given data distribution. The SOM method was first introduced by T. Kohonen (for a summary, see Bishop, 1995; Kohonen, 1997; Vesanto, in press) and can be viewed as a nonparametric regression technique that converts multidimensional data spaces into lower dimensional abstractions.

\section{The Kohonen Algorithm and Self-Organizing Maps}

The original motivation behind the development of self-organizing neural nets was the modeling of basic information processes in the cortex as they are known from neurophysiological experiments. During self-organizing processes, individual neurons become sensitive to specific patterns of sensorial input. Consequently, the synaptic connections among neurons adapt accordingly, so that neighboring neurons "learn" to process similar signal patterns. The Kohonen algorithm defines that relation between the input and the synaptic adaptation of cortex neurons and 
has been successfully proven in discrete simulation experiments with neuron nets of more than 1,000 "biological" elements (for more details on the algorithm described below, we refer to Kohonen, 1997).

A Kohonen net (an $S O M$ ) adapts itself on the basis of the Kohonen algorithm. In most SOM implementations, the SOM is realized by a two-dimensional (often hexagonal) grid. Starting from a set of numerical, multivariate data, the nodes on the grid gradually adapt their weight vectors, $\vec{w}$, to the intrinsic shape of the data distribution. The Kohonen algorithm enables these weights to adapt themselves in response to the input signals, $\vec{x}$, driven by the nodal responses defined as the distance $|\vec{w}-\vec{x}|$. This lets the nodes compete with each other; the closer the weight vector $\vec{w}$ of a node is to the input vector $\vec{x}$, the greater the response. Learning now consists in the fact that the winner node with index $c$ changes its weight vector $\vec{w}_{c}$ to become more similar to the input vector $\vec{x}$. Also, all the neighbors of $c$ within a predefined distance change their weight vectors toward the direction of $\vec{x}$, proportional to the difference between the input vector $\vec{x}$ and the corresponding weight vector (this proportionality factor $\alpha$ is called the learning rate).

After a number of learning steps, the weight vectors of neighboring nodes become more and more similar. Consequently, the Kohonen net is ordered. Since this order on the grid reflects the neighborhood within the data, attributes and features of the data distribution can be read off from the landscape of weight vectors on the grid. Thus, the trained SOM may be seen as a nonlinear representation of the underlying data distribution.

The learning process operates with a number of nodes, which are initially placed in a two-dimensional plane. Like an elastic membrane, the Kohonen net has an intrinsic tension; its nodes attract the currently nearest data vectors to form the plane to a sculptured surface in the data space until the net stretches toward the data clusters to find the best match for the data distribution. The final locations of the nodes correspond to the data distribution in the data space; that means that the number of nodes in a certain region reflects the density of data.

Evaluation of the trained Kohonen net. The weight vectors of a trained Kohonen map can systematically be converted to visual information in order to enable application of a number of evaluation techniques. This is mostly achieved with coloring and shading techniques. Also, the distances between the weight vectors can be evaluated systematically. The weight vectors of several neighboring nodes may form a dense and connected data region (cluster) or may differ significantly from each other (if the corresponding data regions are far apart). Viscovery SOMine automatically draws boundaries and assigns different colors to different clusters. Each component of the weight vectors can be visualized in the entire map and can be compared with other components. Because of the clear presentation of the extracted information, the identification of dependencies between parameters, predictions, cluster analyses, classifications, or the monitoring of system states, to mention a few, becomes an intuitive and stimulating interactive process.

SOM tools such as Viscovery SOMine have been shown to be helpful in analyzing behavioral responses, in biochemical studies and medical diagnoses (e.g., Roberts \& Tarassenko, 1992). For other applications - for example, evaluations of market surveys, customer profiling, market segmentation, customer scoring-see Deboeck and Kohonen, 1998, for techniques, such as pattern recognition (e.g., Chi \& Yan, 1995).

Although all of the improvements described above are potentially intrinsic to the SOM algorithm as such, Viscovery SOMine is recognized to be a tool that includes the described features in a user-friendly way and in a state-ofthe-art implementation. In order to use Viscovery SOMine, there is no need to be familiar with the details of the basic algorithm, the network topology, or weight adjustments, as is usually the case with supervised neural networks. The user is guided through the data import and adaptation process by empirically defined default settings and masks (of course, more advanced uscrs are free to define certain SOM parameters themselves). The same applies to data preprocessing, where specific changes can easily be made through the graphical user interface. Also, the speed of the learning processes has been increased by implementing several enhancements to the original algorithm. Viscovery SOMine is available as Version 2.1 under Windows NT 4.0 and Windows $95 / 98$ at a cost of $\$ 695$ for a singleuser scientific license (for a free demo version, see http:// www.eudaptics.com).

\section{SAMPLE EXPERIMENT}

\section{The Functions of Ocelli for the Flight Control of Giant Honeybees}

The visual system of most flying insects consists of two compound eyes and three ocelli (Götze, 1927). The major feature of the functional design of ocelli has to do with detecting rapid changes (Milde, 1987) in lowintensity patterns summed over the whole visual field (for a summary, see Mizunami, 1994). Ocelli underfocus (Schuppe \& Hengstenberg, 1993) and, therefore, cannot image; they are evidently responsible for two aspects of behavior: minimizing phototaxis (Kastberger, 1990a, 1990b, 1992; Kastberger \& Schumann, 1993) and keeping the flight stabilized through horizon detection (Taylor, 1981; Wilson, 1978). There are, however, arguments that do not support the assumption that these two aspects are all-decisive for the emergence of ocelli in the course of evolution: First, the compound eyes might have done both jobs; second, it holds true, in particular for honeybees, that exposure to phototaxis-inducing stimuli, as well as to a horizon pattern, is not a compulsory feature of every flight event. Therefore, we propose that the continuous changes in illumination that flying insects are exposed to are the more general sensory paradigm that prompted ocelli, in the course of evolution, to serve as an additional flight control system. We tested free-flying 


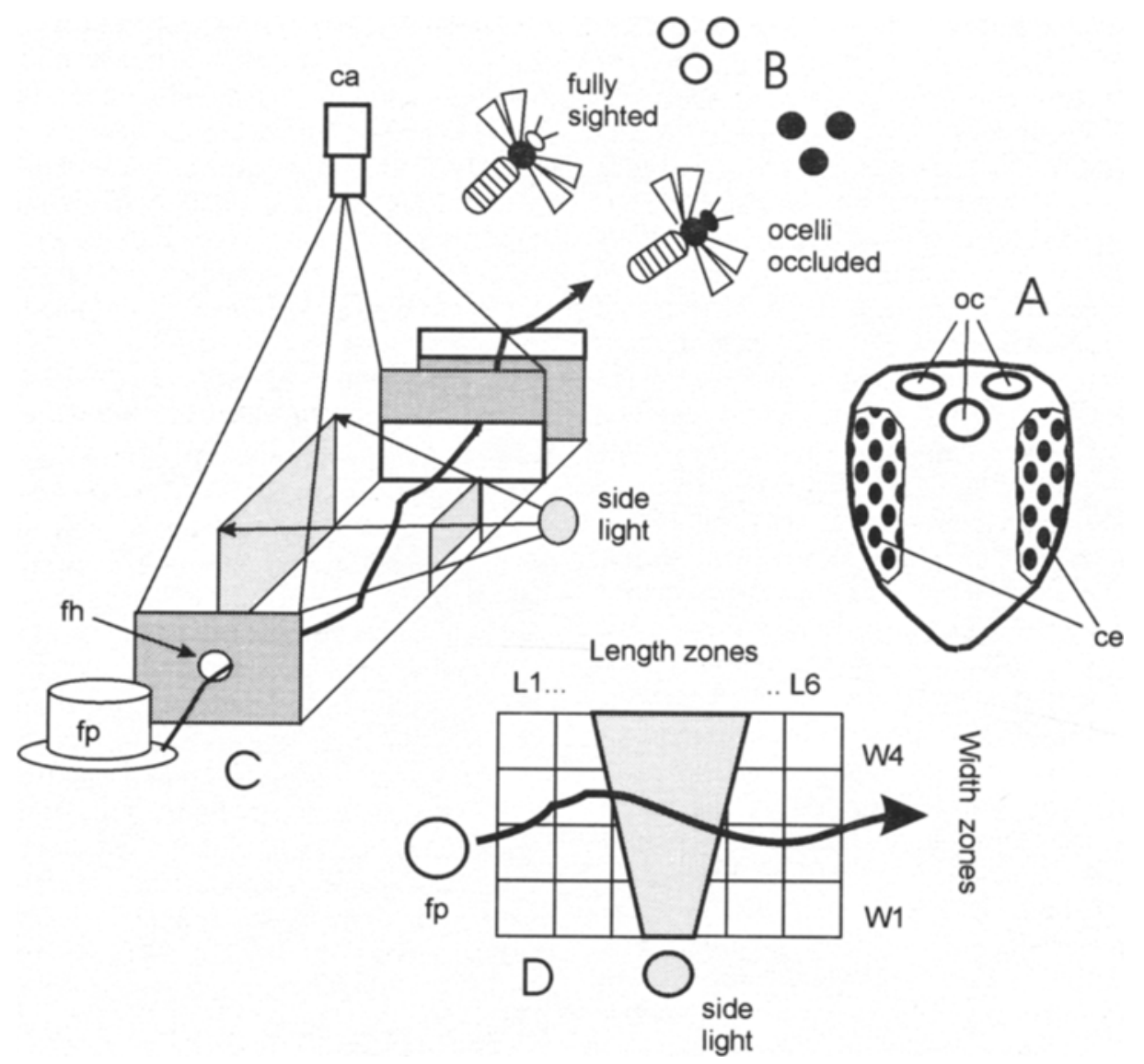

Figure 1. Experimental setup and conditions. A: bee's front view, with two compound eyes (ce) and three ocelli (oc). B: Forager bees tested were marked by color labels on the thorax (fully sighted, yellow; ocelli occluded, green). C: sketch of the flight chamber which the bees had to fly through to go to the feeding hole (fh) and feeding place (fp) or to go home to nest; the sidelight was stationary in states on or off. D: The ground area of the flight chamber was divided into four width $(W 1 \ldots$ $W 4)$ and six length $(L 1 \ldots L 6)$ zones.

giant honeybees (Apis dorsata) and trained them to fly through a chamber in which spatial cues with defined illumination gradients had been established. The aim of this experiment was to find and display adaptive advantages in free-flight orienting that fully sighted bees gain from the ocellar system.

\section{Measurement of Flight Behavior and Ocellar Functions}

\section{Labeling and Occlusion of Ocelli}

Giant honeybee foragers were trained to go from their nest (in the campus of Jawaharlal Nehru University in New Delhi) to a feeder (Figure 1) in the laboratory. The dorsal side of the thorax of 100 forager bees was marked with paint; 50 marked bees were removed from the feeder, and their ocelli were occluded with green nontransparent paint (for further methodological remarks, see Kastberger \& Schumann, 1993). Therefore, two categories of labeled individuals with differing ocellar states were tested (fully sighted bees: compound eyes and all three ocelli untreated; ocelli occluded: untreated compound eyes, all three ocelli occluded).

\section{Observation of Free-Flying \\ Bees in a Flight Chamber}

On their way to the feeder (feeding flight) and back to the nest (homing flight), the bees had to pass a flight chamber measuring $52 \times 32 \times 150 \mathrm{~cm}$ (width $\times$ height $\times$ length). It was illuminated by light coming in through the window entrance and by room illumination. This created an ambient light intensity, which in the mid-zone of the chamber, was 1.500 lux from the top and 100 lux from the bottom. An additional sidelight was introduced to provide a disturbing stimulus to the bee in the flight chamber. This was a normal bulb with daylight characteristics and white waxed paper serving as a dispersing filter in front of it. It was installed at one side of the chamber to produce a wide-field light beam of 5.000 lux into the middle part (as compared with 100 lux when the sidelight was switched to off). 


\section{Assessing Flight Behaviors}

In the chamber, a camcorder tracked from above the experimental bees and identified them by acoustical comments as to their group labels (fully sighted, ocelli occluded). A total of 40,000 positional data of 1,500 flights were evaluated, which were put in relation to two flight targets (feeding and homing flight), two ocellar states ( fully sighted and ocelli occluded), and two stimulus regimes (sidelight on or off). The individual flights were traced from video recordings frame by frame. The coordinates of the thorax and the angle of the long axis of the bee $(L)$ were measured in relation to its position and orientation in the chamber (the coordinates gave the position of the bee in their projection onto the horizontal middle layer, where most of the trained bees flew). The long axis of the chamber was scaled 0 at the side at which the bee had entered. The transverse position was 0 at midline, on the connecting line between the outlet hole near the window and the feeding place on the rear side of the flight chamber (Figure 1). The flight course angle $C$ and the yawing angle $(L c)$, as well as the turning tendencies around the yaw axis $(d L / d t)$ and the flight course $(d C / d t)$, were calculated from successively paired flight data. The sign of the bee's transverse position and of its directional parameters $(L, C, d L / d t, d C / d t)$ were defined by the geometrical relation of the bee to the sidelight: Positive positions were obtained in the half of the chamber on the side of the stimulatory light, and negative positions in the other half of the chamber. Similarly, there were positive angles, and turning tendencies resulted when the bees headed or turned toward the wall with the sidelight. Finally, kinetic aspects were computed, such as ground speed $(V g)$ and ground acceleration $(A g)$.

\section{Flight Chamber Matrix}

The behavioral data were assessed in the flight chamber regarding sampling fields of $160 \times 300 \mathrm{~mm}$ (width $\times$ length) size. They were defined by the coordinates of their centers, which divided the floor of the flight chamber (Figure 1) into four width zones $(W 1 \ldots W 4)$ and six length zones $(L 1 \ldots L 6)$. Since the sampling fields overlapped each other, the behavioral data referred to this matrix were smoothed down in a manner similar to the principle of the running average.

\section{Net Responses}

If a bee entered the flight chamber, it had to decide how to pass along it. The flight path depended not only on the disturbance by the sidelight, but also on the flight chamber conditions in general. Therefore, the proper responses of the bees tested under the sidelight on regime were hidden in the complex interactions of the bees with the flight chamber and could be calculated by subtracting the reference data assessed under the regime with the sidelight off from the data under the regime with the sidelight on. These net responses were computed with respect to ocellar state (fully sighted and ocelli occluded), to target (feeding or homing flight), and to each of the width and length zones; finally, they were checked for significance (less or greater than 0 ) by a chi-squared test. In the following, this computation is explained with the flight parameter $L: m L O N$ and $m L O F F$ are the mean values under sidelight on and off; the differences $(D m L=m L O N-m L O F F)$ represent the net values under sidelight on; and $p D m L$ maps the significance levels of this net effect (for a definition of significance coding, see Table 1 and the Format Requirements of Data Sets section).

\section{Data Mining of Sample Results With Viscovery SOMine}

\section{Format Requirements of the Data Set}

The data source of the sample experiment is stored in Microsoft Excel Workbooks format or in flat files (Table 1). Each data row represents exactly one observational category, which is coded by metric values, since this is necessary for the application of the SOM algorithm.

The parameters assembled in the columns consist of, first, the individual-specific factors of the experimental paradigm: the flight target, coded as $f h=1$ for the feeding flight and as $f h=2$ for the homing flight; and the ocellar state, coded as $o c=1$ for fully sighted bees and as $o c=2$ for ocelli-occluded bees. The second set of columns codes the external experimental factors - that is, the positional information of the bees flying in the chamber relative to the respective width $(w=1 \ldots 4)$ and length $(l=1 \ldots 6)$ zones; the variable $r$ Stim, which codes the probability of exposure of the bees to the sidelight in a chamber area of a given width and length and ranges from $0 \%$ to $100 \%$; and the number of respective observations under the sidelight on and off conditions. Third, we considered the directional and kinetical flight components, as described above, as behavioral measures. We distinguished between sidelight on and off aspects: the sidelight was switched on to establish disturbed conditions; the behavioral patterns under sidelight off are the undisturbed reference. Thus, the behavioral data of each flight parameter consist of on, off and net responses in terms of means and standard deviations, as well as the codings of significance $(0 \ldots p<.10 ;+1,-1 \ldots p<.05 ;+2,-2 \ldots p<.02$; $+3,-3 \ldots p<.01 ;+4,-4 \ldots p<.001)$. Their signs are defined by the net responses in the following way: positive means that the on value is larger than the off value; negative means that the off value is larger than the on value. Table 1 shows only those behavioral components that are related to the flight parameter $L$ - that is, the direction of the length axis of the body of the free-flying bee.

\section{Data Exploration}

The basic question in our sample investigation refers to obvious dependencies of the flight behavior on the experimental conditions. In other words, is the flight behavior of the bees passing through the flight chamber influenced by the flight target, the ocellar state, the position in the flight chamber, or the sidelight disturbance? The Viscovery SOMine data mining tool offers at least two complementary prospects for analyzing such dependen- 
Table 1

Sample Source File for Viscovery SOMine: Flight Data of Fully Sighted and Ocelli-Occluded Giant Honeybees in a Sidelight Disturbance Paradigm and Coding of Experimental Conditions and Flight Behaviors

Experimental conditions

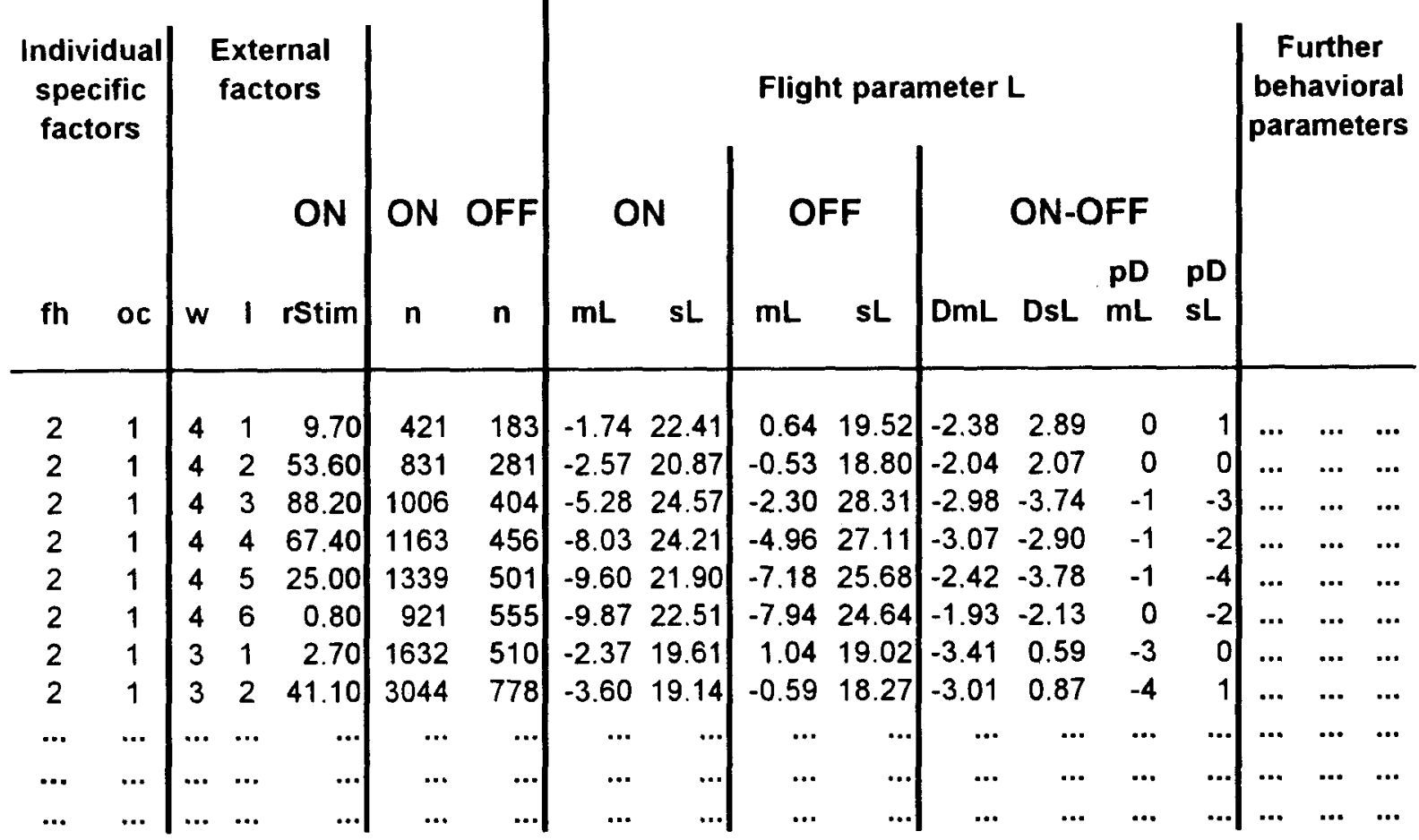

Note--The sidelight was switched on to establish disturbed conditions; the behavioral patterns under sidelight off are the undisturbed references. As behavior, only the direction of the body's length axis $(L)$ of the bee during flight is shown, supplying various components: $m L, s L$ : mean and standard deviation of $L$ direction; $D m L$, $D s L$ : net responses of means and standard deviations; $p D m L, p D s \mathrm{~L}$ : significance level of net responses (see the text).

cies. The basic step of data mining itself is to look for clustering in the behavioral aspects and to compare these clusters in a retrograde way with the experimental conditions. This is achieved by starting the ordering process with the behavioral components as the master and the experimental conditions as the slave criteria. This method is especially useful for the initial exploration of unknown data sets (see the Basic Data Exploration section). A more advanced method, which we used for dependency analyses, is to focus on the shape of behavioral components when they are associated with a prefixed topology of experimental conditions (see the Creating a Defined Topology of Experimental Conditions section, below).

\section{Basic Data Exploration}

Retrograde shaping of the experimental components. The arrangement of the data in Table 1, with its columns as components and its rows as data records, allows us to start the Viscovery SOMine training process directly (note: Viscovery SOMine accepts only a singleheader row containing the names of the parameters) to create a SOM with a two-dimensional hexagonal grid rep- resenting the data distribution (see the Kohonen Algorithim and Self-Organizing Maps section).

The mappings of Figure 2 are the result of such a training process and refer to the whole data set of fully sighted and ocelli-occluded bees on homing and feeding flights. The mappings identify the individual-specific factors of flight target (feeding or homing flight) and ocellar state (fully sighted or ocelli occluded) and the external factor of the position in the flight chamber (width and length zones). This map was created with a special setting of priority, which controls the "importance" of respective components: It can be chosen to internally extend or squeeze these components, which thus have a stronger or weaker impact on the ordering process (e.g., setting priority for a component at $<0.1$ will practically "suppress" this component for the SOM ordering process). In the SOM of Figure 3, priority for the experimental conditions, such as target, ocellar state, and position in the flight chamber, was set at a low value $(0.01)$, whereas priority for all the behavioral components, such as the direction of the length axis of the body, the flight course, or the ground velocity, was set at a high value (1.0). This meant that the 


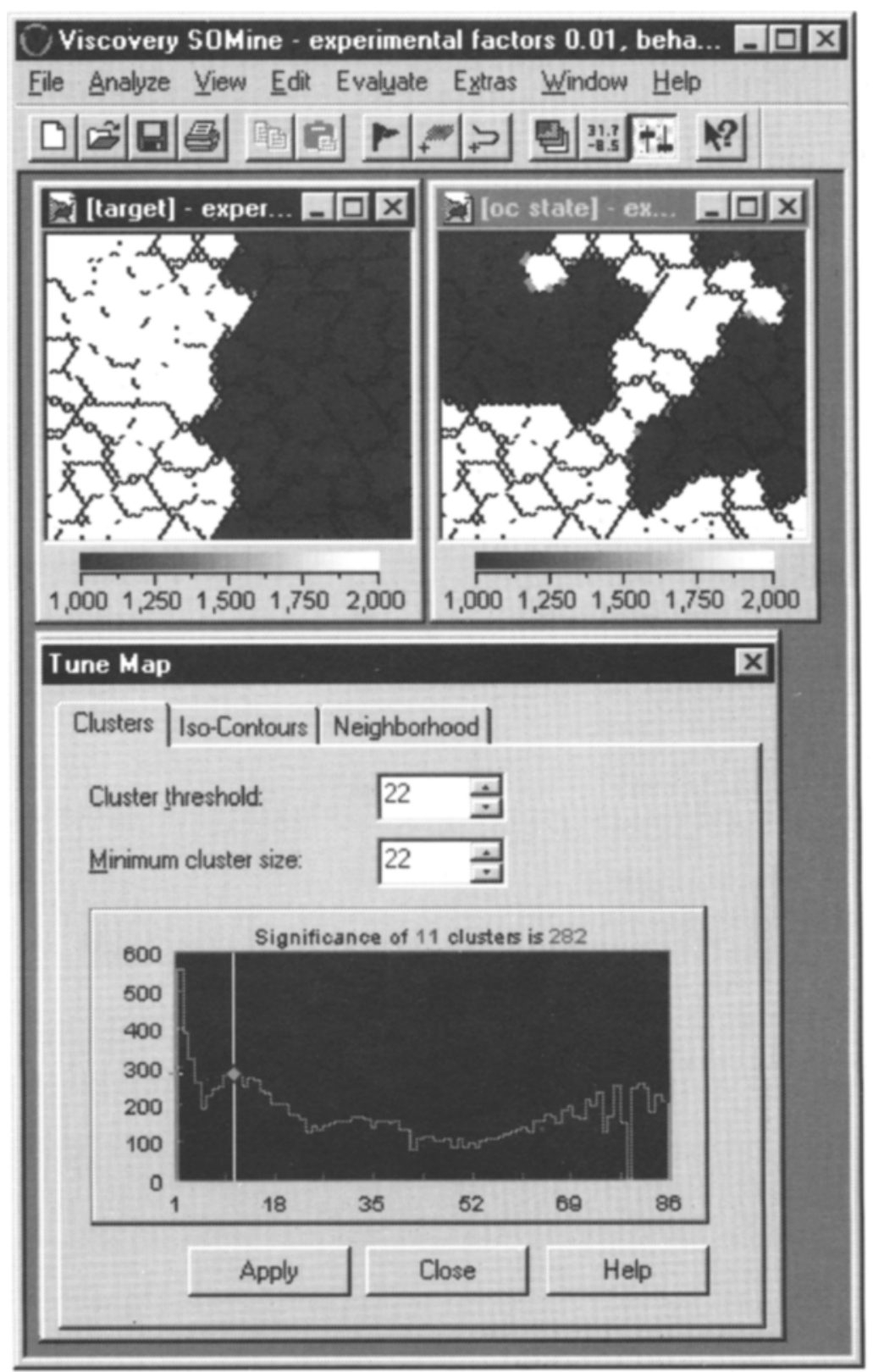

Figure 2. Some main features of the Viscovery SOMine window. The toolbar, which is displayed below the menu bar, provides quick mouse access to important tools (such as creating a new map, applying special evaluations, or switching to the Help mode). In the component windows, selected components are mapped; the active map (here, the left one, target) is highlighted. Each component window shows a readjustable scale bar. As an example, the two windows show the individual-specific factors of the experimental conditions, target and ocellar state. The tune map dialog below can be opened by choosing the menu item analyze and allows for interactive control of cluster separation and display of contours of similarity and neighborhoods.

grid order was determined mostly by the behavioral aspects of the data and less by the experimental conditions.

Viewing the intrinsic shape of behavioral data. The component windows of the map resulting from the SOM training process directly display the intrinsic shape of the nonlinear relations within the data. Dependencies between components can be viewed if the component windows are placed side by side. As is common in statistical analyses, scaling was normalized to variance (however, in Viscovery SOMine, priority is an additional scaling factor). The gray- or color-scaled map regions (for this black-andwhite reproduction we used gray-scaled mappings) represent different clusters. The clusters divide the input data into disjoint areas containing similar vectors. The gran- 

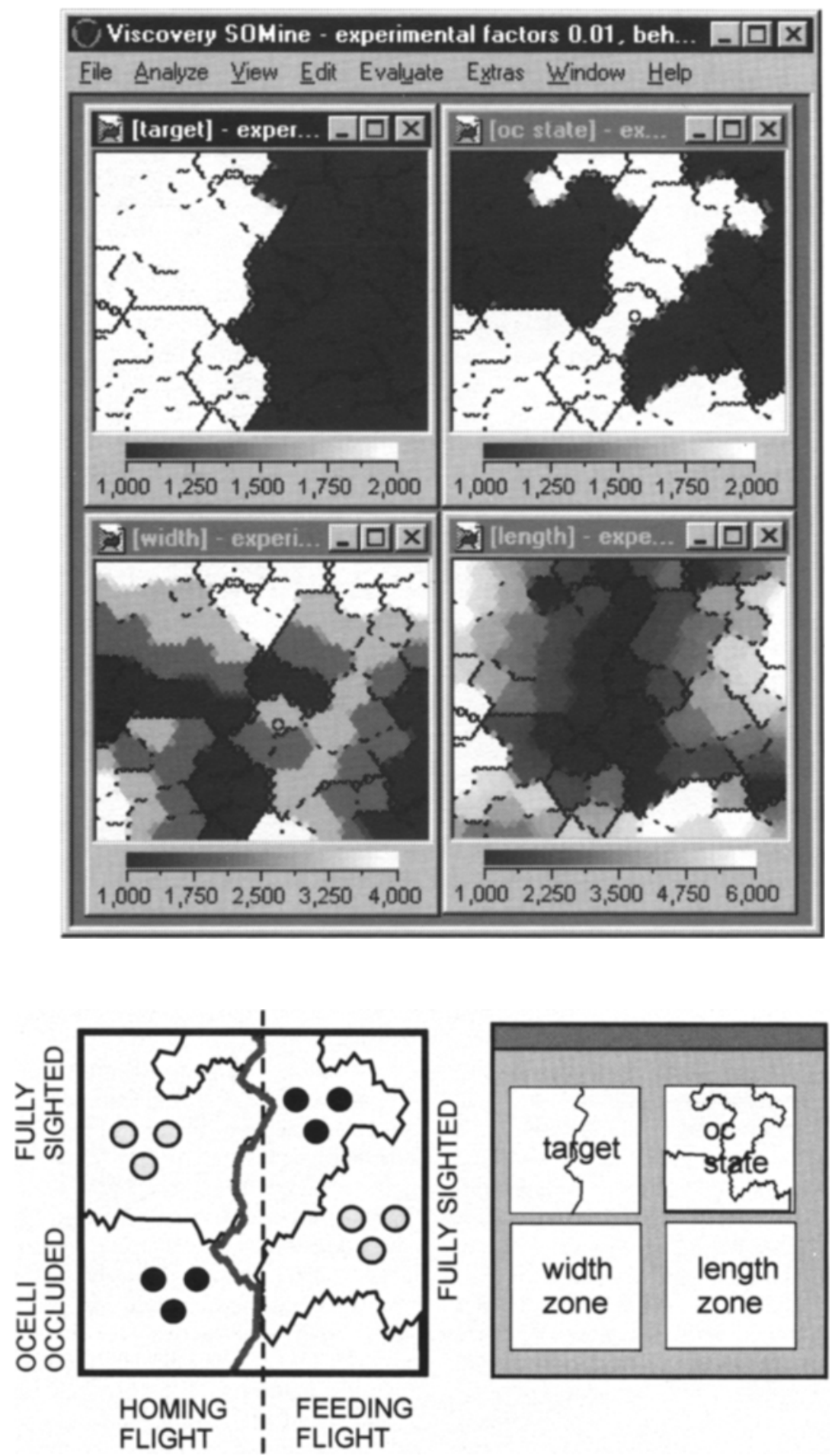

Figure 3. Viscovery SOMine screenshot, which displays four maps regarding the individualspecific (flight target, ocellar state) and external (geometrical position as width and length zones) factors of experimental conditions; the priority factor for experimental conditions was set at 0.01 and for behavioral components (not shown here) at 1.0. Black lines inside the component windows show the cluster separators. The sketch at the bottom on the left summarizes the topology of the component windows with respect to both individual-specific factors; the sketch on the right denominates the four windows of the screen shot. 
ularity of the clustering may be controlled by choosing appropriate parameters in the tune map dialog so that different data regions can be interpreted in a meaningful way. The quality measure for each cluster count is indicated by the internally defined cluster indicator (see Figure 2). If the latter is high, the clustering may be viewed as "natural" for the map; if the significance is low for a cluster count, that clustering may be viewed as "artificial." This signifies that the intrinsic shape of the data does not reflect expected relations.

For example, in the case of Figures 2 and 3, the map pattern was best described by 12 clusters at a cluster indicator of 261 , at a cluster threshold of 22 , and at a minimum cluster size of 17 nodes.

Interpretation of clustering. Owing to their low priority level, the components displayed in Figures 2 and 3 did not contribute much to the ordering process (only the behavioral components were used for SOM training). Nevertheless, the maps show a distinct, heterogeneous distribution for the individual-specific factors (see the black and white areas of flight target and ocellar state) and, also, for the components of the geometrical position (width and length zones); the opposite case would exist if the black, gray, and white areas were randomly dispersed. Therefore, the maps in Figures 2 and 3 reveal the important influence of the experimental conditions on flight behavior.

\section{Advanced Exploration}

Shaping behavioral components with a prefixed topology. In the following specific case, we determined a prefixed order in which to create the SOM. Such a procedure may help to trace dependencies in data distributions when there is already a hypothesis on the subject. This reflects the broadly used principle of verifying that a response (slave event) is caused by a stimulus (master event). In the case of the sample data set of flight behavior (Table 1), we want to show how the behavioral data align into a given topology of experimental factors. If the behavioral data (given a low-order priority level) reflect the topology of the experimental factors (given a highorder priority level), such as flight target, ocellar state, or the geometry of the flight chamber (width and length zones), proof is furnished that these experimental factors have an impact on the behavior. No dependency exists if the maps of the behavioral components show a uniform or randomly structured pattern. Not essentially, but practically, this kind of exploration offers a better access to the paradigm-specific responses of the experimental animals than do the earlier mappings (Figures 2 and 3 ). The behavioral effects in Figures 4 and 5 are adapted to a more geometrical order of the experimental components (the mapping of the component target in Figure 3 seems to be similar to the respective map in Figure 5, but the intrinsic order is well demonstrated by the experimental components width and length).

Creating a defined topology of experimental conditions. In Figure 4, the data set was presented to the training process with the following priority settings: A high impact (priority was set as 10) was defined for both of the individual-specific factors of flight target and ocellar state, and a slightly lower impact ( priority was set as 5) was defined for the geometrical factors (width and length zones), in order to create a basic topology of the data set in the following format. In each component map, the data of the fully sighted bees were arranged in the bottom clusters; those of the ocelli-occluded bees were arranged in the top clusters. Both areas of the ocellar states were similarly sized and shaped (rectangles of similar size), because the data sets of both ocellar states were equal in number (Table 1). Note that the size of the data set was, in this case, determined only by the experimental conditions (flight target, ocellar state, position in flight chamber) and not by the number of observations (which are separate components in the mapping: see map $N f s$ in Figure 4 for an example of observations of fully sighted bees). Similarly, the data of feeding flights are placed at the right side of the map, that of homing flights at the left side. Furthermore, the components representing the flight chamber geometry (width and length zones) are nested into each of the four primary clusters, because their priority was lower than that of the individual-specific factors.

One of the advanced techniques that Viscovery SOMine offers for data exploration is that it associates values with data records, which means that additional components can be attached to the map without influencing the training process. We set the priority of these components (such as the number of observations and the rate of exposure to the sidelight, as well as the behavioral aspects), which are to be associated with the remaining components, to a very small value, below 0.1 (see also the Retrograde Shaping of the Experimental Components section for information about priority).

Displaying the stimulus condition of sidelight disturbance. The map rStim (on the right side in Figure 4) is a visualization of the rate of exposure to the sidelight, a further external factor for all four bee categories (fully sighted and ocelli-occluded bees on feeding and homing flights) that pass through the flight chamber. It should have no influence on the ordering process. These values range from $0 \%$ (coded here as black areas; in default rainbow color scaling, it would be coded as blue) to $100 \%$ (coded here as white areas; red, in default rainbow color scaling). The pattern of this component reflects the disturbing conditions for the bees and agrees well with the geometry of the nonparallel, wide-field beam of the sidelight (e.g., in Width Zone 4, the area opposite to the wall with the sidelight wall, the probability of being exposed to the sidelight is highest).

Sample mappings of directional and nondirectional flight behaviors. In Figure 5, six flight parameters are used to explain and visualize complex behaviors. The SOMs have the same prefixed topology of experimental conditions as that shown in Figure 4. Each map has four submaps: Homing bees are represented at the 

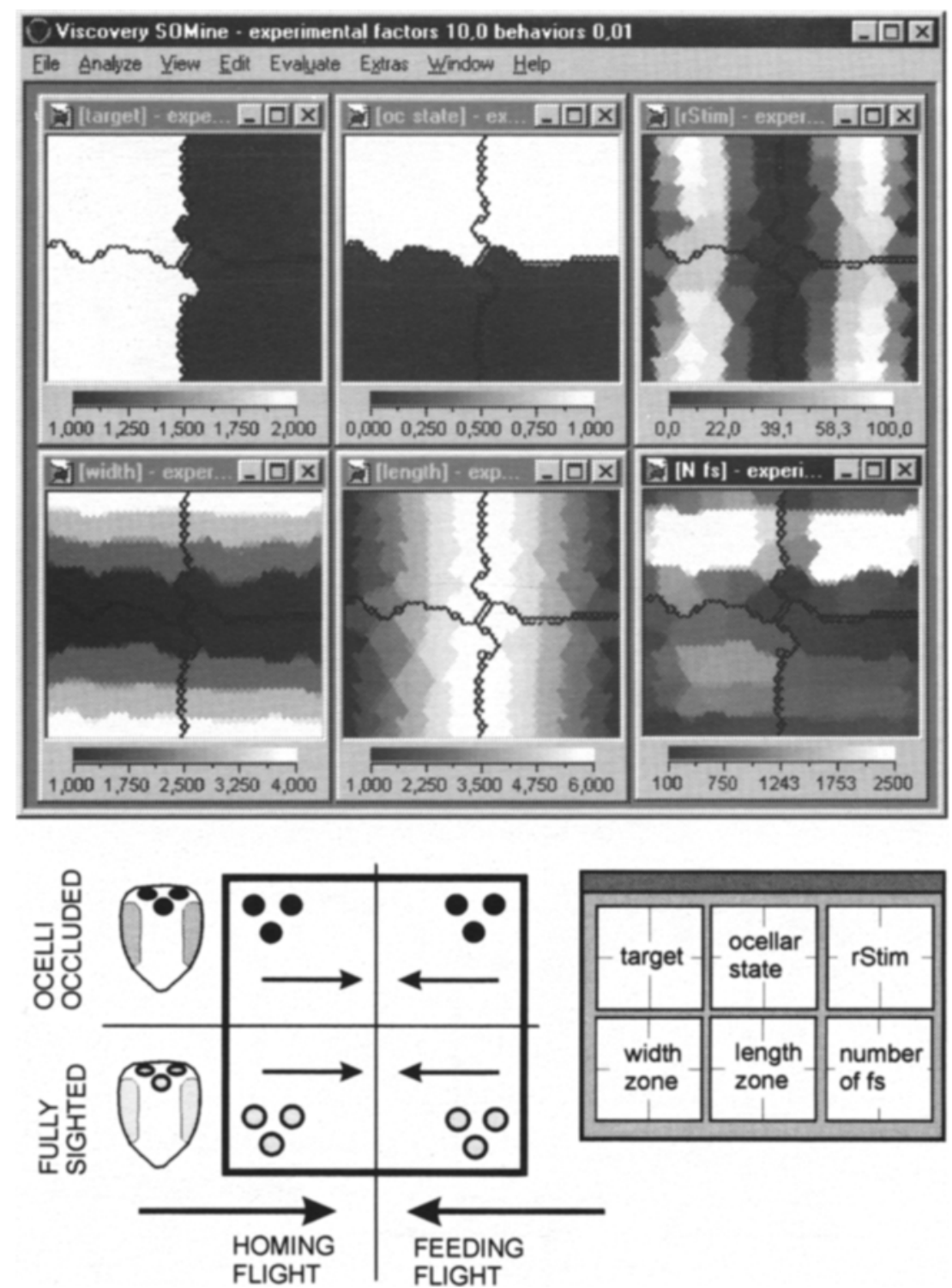

Figure 4. Mapping the individual-specific and external factors of experimental conditions; the priority of target and ocellar state was 10, that of geometrical position (width and length zones) was 5 , whereas the behavioral components (rStim, the rate of exposition to the sidelight, and $N f s$, the number of observations regarding fully sighted bees [scaled from 100 to $2,500])$ was associated with the experimental conditions under a low priority $(0.01)$. The sketch at the bottom on the left summarizes the topology of the maps with respect to both of the individual-specific factors, flight target and ocellar state; the sketch on the right denominates the six maps of the screen shot.

left, bees on a feeding flight are on the right; the lower submaps refer to the fully sighted state, the upper ones to the ocelli-occluded state. All the component windows reflect the significance coding of the net response, the way the sidelight disturbance affects the respective behaviors of free-flying bees: The white areas display a significant $(p<.001)$ positive effect, the black areas a significant $(p<.001)$ negative effect of sidelight on. In the directional parameters, the sign of the responses is additionally joined to the direction in which the flying bee tested in the flight channel turns: Positive responses refer to turning to the sidelight wall; negative responses refer to turning to the wall opposite the sidelight (see also the Measurement of Flight Behavior and Ocellar Functions section and the Creating a Defined Topology of Experimental Conditions section).

\section{What Can Be Viewed}

The direction of the body's length axis. The component window $p D m L$ (Figure 5, left top) displays homing 

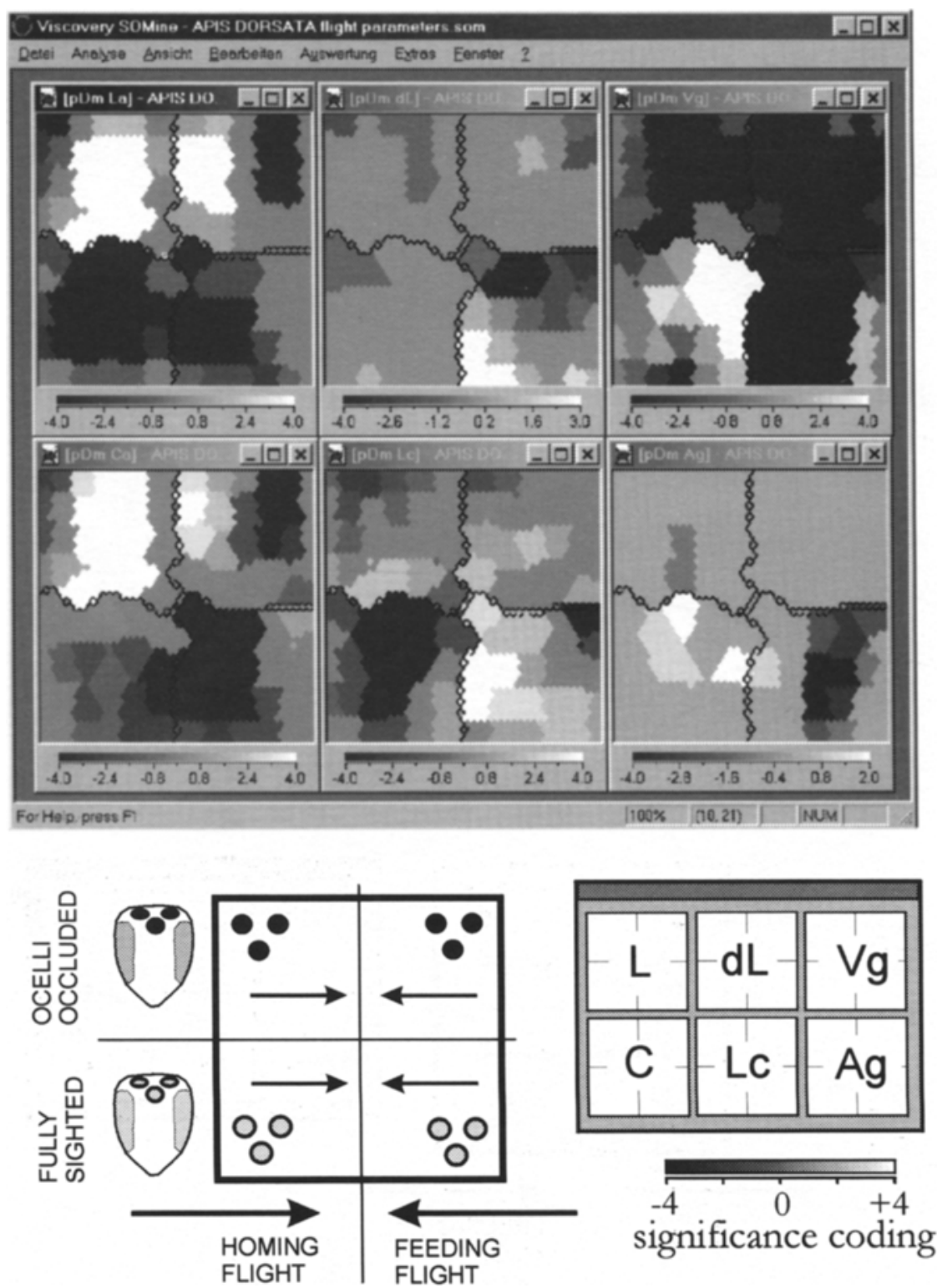

Figure 5. Viscovery SOMine screen shot of six component windows $(m X)$ with respect to $L$ (the direction of the length axis of the body of the free-flying bee), $C$ (the flight course), $L c$ (the yawing, $L-C$ ), $d L$ (the turning tendency, $d L / d t), V g$ (the ground velocity), and $A g$ (ground acceleration). $D m X(=m X O N-m X O F F)$ represents the net value under the sidelight on condition (see also the Measurement of Flight Behavior and Ocellar Functions section). All six SOMs refer to significance coding ( $p D m X$ ) of this net effect (for $a$ definition, see Table 1 and the Format Requirements of Data Set section). Most of the maps are scaled between -4 and +4 ; only $p D m d L$ and $p D m A g$ are scaled from -4 to +3 and +2 , respectively. The priority for all six components was set to 0.01 (compare Figure 4). The sketch at the bottom on the left summarizes the topology of the map with respect to both individual-specific factors, flight target and ocellar state; the sketch on the right denominates the four windows of the screen shot.

bees as being negatively oriented; this means that those bees turn more to the wall opposite the sidelight. The absolute data (not mapped here) shows that fully sighted bees fly well aligned with the main axis of the flight chamber, because they keep their bodies only at a small but significant angles off the sidelight (around $-2^{\circ}$ ). Ocelli-occluded bees show a marked difference to fully sighted bees. They strongly turn positively to the sidelight (up to $+30^{\circ}$, not shown here). Their turning is determined by the distance to the sidelight wall and is greatest near the sidelight (at Width Zone 1; see also Figure 4). As might be expected, such positive behavior of ocelli-occluded bees did not occur when the disturbing sidelight was switched off (not shown here). Thus, the net 
effect with sidelight disturbance displays marked differences between fully sighted and ocelli-occluded individuals under both flight target conditions. Ocelli-occluded bees not only have a strongly positive phototactical reaction, they also are disturbed by the sidelight in a different way when they are on their way to the feeder than when they are flying homeward. On a homing flight, when they are fully exposed to the sidelight, they turn toward $i t$, whereas on a feeding flight, they turn negatively when they enter the beam and turn positively after they have crossed it.

The flight course. The patterning of the flight course component $p D m C$ (Figure 5, left side, bottom) resembles that of the direction of the body's length axis, $p D m L$.

Comparison of both directional flight behaviors, $\boldsymbol{L}$ and $\boldsymbol{C}$. The patterns of both directional flight components - the direction of the length axis of the body, $L$, and the flight course, $C$-are similar if we compare both behaviors in terms of mean net responses, coded by their significance levels. However, mapping the yawing response, $L c$ (which is the difference between $L$ and $C$ ), further shows (Figure 5, midcolumn, bottom map) a considerable difference between the fully sighted and the ocelli-occluded states: Ocelli-occluded bees exhibit only weak yawing effects; fully sighted bees show quite different patterns in feeding and homing flights.

The turning tendency as an indicator with which to analyze the behavioral strategy of centering. The turning tendency is displayed by the component $p D m d L$ (Figure 5, midcolumn, top map) and refers to circular speed around the body's vertical axis (yaw axis). Mapping this behavioral component demonstrates how behavior is formed as a strategy, in the sense of a responsiveness to spatial cues. At first sight, the patterns under the sidelight on and off conditions are very similar: The bees generally turn positively - that is, toward the sidelight wall - when they fly in Width Zone 4 and turn negatively, toward the wall opposite the sidelight, in Width Zone 1 (not shown in Figure 5). This behavioral pattern is known as the orienting strategy called centering behavior and is practically independent of both the state of disturbance (sidelight on and off) and the individual-specific factors (flight target and ocellar state). This result was expected, because centering behavior requires imaging abilities of visual control, which can only be performed by compound eyes. But these remained unaffected when the ocelli were occluded.

However, the net responsiveness to sidelight disturbance reveals effects that significantly contrast fully sighted and ocelli-occluded bees; in this map, at least for feeding flight conditions, it is proven that fully sighted bees again reveal centering behavior, whereas ocelli-occluded bees exhibit a further residual phototactical responsiveness.

Nondirectional behaviors: Ground speed and ground acceleration. The bees showed different flight speeds when they were flying to the feeder or homeward (not shown in the map in Figure 5). This was to be expected, because homing bees were generally slower after their start from the feeder than were bees that, on entering the chamber, slowed down on their fast flight from the nest. Ocelli-occluded bees generally flew slower than fully sighted bees under undisturbed conditions (under sidelight off; not shown here) and further decreased their speed under disturbing conditions ( $p D \mathrm{mVg}$ in Figure 5, right side, top map). Fully sighted bees behaved differently when disturbed: They sped up on homing flights and lowered their speed when flying to the feeder. The respective effect of acceleration or deceleration can also be observed in the map $p D m A g$ (Figure 5, right side, bottom map).

\section{Interpretation of the Findings}

1. The ocellar system minimizes phototaxis and makes orientation independent of motivational settings, like those we investigated under the term flight target (bees flying to the feeder or home to their nest). This was shown by both directional parameters - $L$, as a measure for posture during flight, and $C$, the flight course.

2. The directional components, $L$ and $C$, are strongly coupled. However, fully sighted bees are, nevertheless, able to separately control the direction of the body and the flight course, which is particularly visible under sidelight disturbance. The bees remain uninhibited in operating their posture and flight course separately, which obviously matches their different orientational needs with different targets (homing or feeding). Ocelli-occluded bees lack this flexibility; both flight components remain strongly coupled here. In other words, the differences in the map patternings (of the significance-coded net responses) document that the ocelli do mobilize an additional ability to keep the bee's flight posture flexible.

3 . The ocelli enforce additional centering capabilities under disturbed conditions, probably interacting here with the domain of the compound eyes.

4. Bees use different strategies on homing and feeding flights, which is, in particular, reflected in the flight kinetics. Bees that are being disturbed generally reduce their speed. Ocelli are mainly used at higher speeds, at which they enable the individual bees to adjust their speed to their orientational needs and make speed control more precise.

\section{REFERENCES}

Bishop, C. M. (1995). Neural networks for pattern recognition. New York: Oxford University Press.

CHI, Z., \& YAN, H. (1995). Handwritten numeral recognition using a small number of fuzzy rules with optimized defuzzication parameters. Neural Networks, 8, 821-827.

DEBOECK, G., \& KOHONEN, T. (1998). Visual explorations in finance. London: Springer-Verlag.

GöTzE, G. (1927). Untersuchungen an Hymenopteren über das Vorkommen und die Bedeutung der Stirnaugen [Investigation of the occurrence and significance of ocelli in hymenopterans]. Zoologische Jahrbücher (Physiologie), 44, 211-268.

KASTBERGER, G. (1990a). Ocellar course and yaw control in honeybees on feeding flight. Zoologische Jahrbücher (Physiologie), 94, 229-245.

KASTBERGER, G. (1990b). The ocelli control the flight course in honeybees. Physiological Entomology, 15, 337-346.

KASTBERGER, G. (1992). The ocellar control of orienting subsystems in 
homing honeybees, investigated under side-light-switching conditions. Zoologische Jahrbücher (Physiologie), 96, 459-479.

Kastberger, G., \& Schuhmann, K. (1993). Ocellar occlusion effect on the flight behavior of homing honeybees. Journal of Insect Physiology, 39, 589-600.

KOHONEN, T. (1997). Self-organizing maps. Berlin: Springer-Verlag. MiLDE, J. J. (1987). The ocellar system of the honeybee. In R. Menzel \& A. Mercer (Eds.), Neurobiology and behavior of honeybees (pp. 191200). Berlin: Springer-Verlag.

MizUNAMI, M. (1994). Information processing in the insect ocellar system: Comparative approaches to the evolution of visual processing and neural circuits. Advances in Insect Physiology, 25, 152-265.

Roberts, S., \& TARASSENKo, L. (1992). New method of automated sleep quantification. Medical \& Biological Engineering \& Computing, 30 , 509-517.
SCHuPPE, H., \& HeNGSTENBERG, R. (1993). Optical properties of the ocelli of Calliphora erythrocephala and their role in the dorsal light response. Journal of Comparative Physiology A, 173, 143-149.

TAYLOR, C. P. (1981). Contribution of compound eyes and ocelli to steering of locusts of flight: I. Behavioral analysis. Journal of Experimental Biology, 93, 1-18.

VESANTO, J. (in press). SOM-based data visualisation methods: Intelligent data analysis (IDA). Amsterdam: Elsevier.

WILSON, M. (1978). The functional organization of locust ocelli. Journal of Comparative Physiology, 124, 297-316.

(Manuscript received December 18, 1998; revision accepted for publication October 3, 1999.) 